Recherches en didactique des langues et des cultures

Les cahiers de l'Acedle

14-2 | 2017

Dialogisme et discours en situations didactiques

\title{
Dialogisme, discours familiaux et apprentissage du français en classe
}

Florence Guiraud

\section{OpenEdition}

Journals

Édition électronique

URL : http://journals.openedition.org/rdlc/1885

DOI : $10.4000 /$ rdlc. 1885

ISSN : 1958-5772

Éditeur

ACEDLE

Référence électronique

Florence Guiraud, «Dialogisme, discours familiaux et apprentissage du français en classe »,

Recherches en didactique des langues et des cultures [En ligne], 14-2 | 2017, mis en ligne le 15 juin 2017 , consulté le 19 avril 2019. URL : http://journals.openedition.org/rdlc/1885 ; DOI : 10.4000/rdlc.1885

Ce document a été généré automatiquement le 19 avril 2019

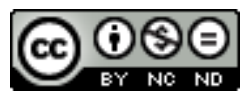

Recherches en didactique des langues et des cultures is licensed under a Creative Commons AttributionNonCommercial-NoDerivatives 4.0 International License 


\title{
Dialogisme, discours familiaux et apprentissage du français en classe
}

\author{
Florence Guiraud
}

\section{Introduction}

1 Nous proposons d'étudier dans le présent article, le discours en situation d'oral conversationnel de jeunes apprenants du français en contexte scolaire, au cours de leurs premiers mois à l'école française. Leur production langagière de niveau A1-A2 se situe encore à l'intérieur d'un espace interlinguistique ou interlangue (Selinker, 1972/2014 : 223) dans lequel ils évoluent en acquisition du français langue seconde (FLS).

Le concept de dialogisme (Bakhtine-Volochinov, 1929/77) a pris une place importante en analyse du discours en France depuis les années 1970. En englobant les notions de mémoire discursive, d'hétérogénéité, d'interdiscours, le principe dialogique permet de poser un paradigme d'analyse qui pose l'altérité discursive et énonciative comme constitutive de tout acte de parole. Mais ce concept a rarement été rapproché de l'analyse de formes verbales relevant de l'interlangue. Or, en définissant tout énoncé comme le résultat d'un réseau de diverses influences linguistiques, le dialogisme met en lumière le rôle déterminant $\mathrm{du}$ contexte social et linguistique dans la production langagière. L'énoncé est marqué, dans sa structure même, par la sphère d'échange dans laquelle il s'est constitué. Ainsi son contenu thématique, sa structure syntaxique, son style, sont le résultat d'un choix sélectif opéré par le locuteur et directement lié à la spécificité de l'environnement social que constitue le contexte de production. La variété de ces sphères d'échanges génère donc des types de discours propres à chaque type de situation. C'est ainsi que Bakhtine définit les genres de discours. Par là même, le dialogue en situation d'oral conversationnel est un type de discours: le discours quotidien. Dans cette perspective, le genre de discours choisi par le sujet-apprenant peut être considéré comme l'élément visible et observable du lien étroit qui unit la production langagière et son milieu. Or la production langagière des élèves apparaît largement animée par des reprises en écho d'autres discours ayant eu lieu en amont et issus d'autres sphères d'échanges. 
Entre ces différentes situations de communication, un fil linguistique semble s'être tissé. Dans un processus dialogique interdiscursif, l'apprenant oriente son discours et rend compte de ces différentes sphères d'échange.

3 Ainsi, chez ces apprenants du français, l'observation au long cours puis l'analyse de leurs énoncés discursifs en situation d'oral conversationnel laissent apparaitre des formes syntaxiques qui semblent marquées par une hétérogénéité énonciative et discursive, notamment au niveau des déictiques (i) et des temps verbaux (ii).

(i) mon père je pars demain: le locuteur et l'énonciateur ne coréfèrent pas; la forme s'apparente à une reformulation ou à du discours rapporté.

(ii) une fois en Italie je travaille bien : le présent est utilisé avec un marqueur temporel passé.

Ces segments discursifs, éloignés syntaxiquement de la norme attendue, pourraient être qualifiés un peu hâtivement d'emplois fautifs, imputables à la non-maîtrise de la langue par les apprenants à ce stade de leur apprentissage.

\section{Dialogisme et espace interlinguistique}

5 Dans cet espace-temps que constitue l'interlangue, l'acquisition de la langue cible est soumise aux influx des langues d'origine dans toutes leurs richesses, c'est là l'une des caractéristiques de l'interlangue.

6 La recherche en didactique des langues a souvent démontré et explicité le rôle et l'influence des langues maternelles sur la construction syntaxique (Selinker, 2014). Mais au-delà de cet aspect, il semblerait que l'apport du cadre théorique du dialogisme permette de représenter cette interlangue plus globalement dans un espace interlinguistique: linguistic space (Selinker, 2014: 223) dans laquelle la production langagière de l'apprenant évolue. Cet espace ou cette distance entre la langue maternelle et la langue cible sont, semble-t-il, marqués par des phénomènes langagiers spécifiques. En effet, l'analyse au niveau micro-syntaxique laisse apparaître que certains segments discursifs paraissent être des éléments énonciatifs, hétérogènes au discours ». (Anscombre, 2006 : 6). Ces éléments se manifestent comme autant de traces qui peuvent être à la fois d'ici et d'ailleurs, constitutives du discours et en même temps étrangères à celui-ci. (ibid.). Cette trace prend la forme d'un segment discursif présent dans l'énoncé effectif du locuteur que nous observons, mais aussi comme ayant appartenu à un autre locuteur dans une autre situation de communication, comme semblent l'attester ici les déictiques.

maîtresse, moi tu t'assois ici?

ma mère, je vais faire une opération à la main.

7 Ces différents énoncés entrent en interaction, s'entrechoquent et se mêlent au sein d'une syntaxe devenue dialogique, Dialogic syntax (Du Bois, 2014).

Ainsi, l'organisation syntaxique de l'énoncé semble manifester une relation dialogique avec un autre cadre que celui de l'échange effectif dans le contexte scolaire. Le cadre dialogique permet de replacer l'énoncé non seulement dans le cadre visible de l'interaction verbale, mais aussi dans son histoire interdiscursive et conversationnelle. Aborder l'analyse de la production langagière des apprenants en mettant en évidence les dialogues internes ou, en d'autres termes, la dimension dialogique de leurs énoncés, permet de formuler certaines hypothèses explicatives aux faits de langue observés. Il semblerait que leurs productions langagières parviennent à se réaliser grâce à la 
rencontre d'autres discours (dialogisme interdiscursif) réalisés dans une autre sphère d'échange. C'est ce que nous tenterons de démontrer dans le présent article.

\section{Méthodologie}

Il s'agit d'étudier des productions langagières d'apprenants du français, dans une situation de dialogue, c'est-à-dire d'échanges pendant le temps scolaire. Le corpus est constitué d'enregistrements audio de classe de FLS en situation d'apprentissage. Le corpus a été réalisé auprès de 31 élèves allophones, nouvellement arrivés en France et âgés de 6 à 12 ans. Il comprend 2 heures d'enregistrement par semaine durant les 5 premiers mois de l'année scolaire, ainsi qu'un corpus vidéo de 15 heures d'enregistrement. Il s'agit donc de données, relevées lors d'une observation au long court. Les langues familiales des sujets observés sont l'arabe dialectal et l'espagnol. Les fonctionnements syntaxiques et lexicaux de ces langues ont été pris en compte dans l'analyse (Ben Amor Ben Hamida, 2009). Ainsi, pour tenter d'étudier ces emplois d'apparence fautifs au niveau (i) des déictiques et (ii) de l'expression de la temporalité chez ces apprenants (voir supra), un relevé sur le vif des différentes interactions langagières paraissait la méthode la plus adéquate. Les enregistrements ont été effectués sur des temps informels hors des séquences d'apprentissage du FLS. Il paraissait essentiel de préserver toute la spontanéité de l'interaction langagière et d'éviter chez l'apprenant un ressenti potentiellement inhibant d'oral surveillé. Pour cela, le microphone n'était pas ostensiblement visible.

Ces échantillons de segments discursifs relevés lors de plusieurs séquences d'échanges conversationnels entre l'apprenant et l'adulte ou entre les apprenants ont été retranscrits. Ils ne sont pas exhaustifs de toutes les interactions rencontrées dans les différents échanges. Ils constituent pour chacun d'eux, un exemple-type des différents emplois apparemment fautifs que l'on a pu relever chez ce type de sujets. Ils sont donc tous issus de l'oral communicationnel, en classe avec l'adulte enseignant ou entre pairs dans le cadre d'une communication exolingue, et serviront de base à l'analyse. Les énoncés produits sont socialement inscrits. Leur contexte de production détermine et fournit les éléments langagiers nécessaires à leurs constructions. Bakhtine rappelle ainsi que l'interaction verbale est la réalité fondamentale de la langue (1929-1977 : 136). C'est ainsi, en observant le contexte global de production de l'énoncé que nous pourrons formuler des hypothèses permettant d'expliquer les emplois d'apparence fautifs.

\section{Analyses}

11 Dans les 3 extraits analysés, le codage $P$ indique que le locuteur est le professeur avec qui se déroule l'entretien. Les prénoms d'origine des apprenants ont été modifiés. Les passages marqués en gras sont ceux qui servent davantage l'analyse.

$\mathrm{P}$ : qu'est-ce que tu voudrais faire plus tard toi Ibrahim après le collège $\uparrow$

Ibrahim : tu continues

$\mathrm{P}:$ hein $\uparrow$

Ibrahim : tu continues et tout / tu vas au lycée // et tout // je travaille /

après je vais // être un joueur 


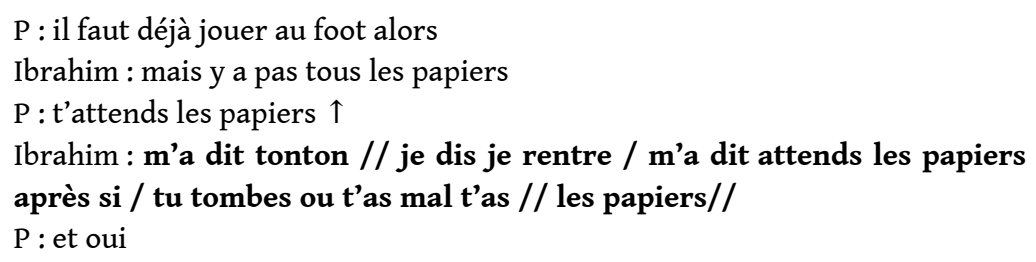

La discussion a lieu lors d'un temps informel: hors séance d'apprentissage, dans une situation d'oral non-surveillé, ce qui peut laisser supposer que l'apprenant se sent moins soumis à des contraintes discursives liées à la position asymétrique élève/professeur. Le thème de la conversation porte sur la situation délicate de l'apprenant quant à son avenir et à sa situation administrative comme migrant en France. Au moment de l'enregistrement, Ibrahim est en France depuis 9 mois. Venant d'Algérie, sa langue d'origine est l'arabe dialectal. Par ailleurs, au moment de l'enregistrement, sa situation administrative n'est pas encore régularisée.

Lorsque nous observons l'énoncé au niveau micro-syntaxique, il apparait que sa composition est affectée par un phénomène langagier spécifique : un écho dialogique évoquant syntaxiquement les formes du discours rapporté.

On en trouve ici plusieurs occurrences. Observons le fragment discursif :

tu continues et tout / tu vas au lycée //et tout

On note ici de la part du locuteur, l'usage fautif du tu pour parler de lui-même. Pour expliquer ce flou référentiel au niveau du pronom déictique $t u$, trois hypothèses s'offrent à nous.

La première consisterait à penser qu'il peut s'agir d'une défaillance linguistique de la part de ce jeune apprenant en France depuis 9 mois seulement. L'utilisation des pronoms personnels est souvent difficile à acquérir chez des sujets dont la langue maternelle est l'arabe dialectal. En effet, l'arabe possède un système pronominal éloigné de celui du français (Ben Amor Ben Hamida, 2009). Cependant, Ibrahim a eu l'occasion de montrer à plusieurs reprises dans ses différents tours de paroles, qu'il ne commettait pas d'erreurs de cet ordre-là. Le segment discursif suivant : je travaille / après je vais. / être un joueur, atteste d'une utilisation tout à fait correcte du pronom personnel sujet je dont le contenu référentiel est déterminé par la situation de communication.

La deuxième hypothèse orienterait l'analyse vers le tu générique ou tu arbitraire. En effet couplé avec l'emploi du présent, la particularité du tu générique est d'ouvrir la place de tu à n'importe quel individu. Ce qui revient à dire que n'importe quel référent peut prendre cette place, dans une situation d'énonciation définie. Or cet emploi spécifique, supposerait de la part de l'apprenant, une maittrise des référents déictiques peu probable à ce stade de son apprentissage.

17 Nous privilégierons donc ici une troisième hypothèse. On peut supposer que cet énoncé : tu continues et tout // tu vas au lycée // et tout, rapporte les paroles d'un autre énonciateur in absentia. En effet, l'utilisation anormale de tu pour parler de lui-même nous invite à penser qu'à ce moment-là la production langagière du locuteur est traversée par un discours antérieur, imputable à une autre instance énonciative qu'il livre ainsi telle quelle. Le discours laisse donc entendre deux voix, imbriquées l'une à l'autre de façon confuse dans un même énoncé. En effet, malgré l'absence d'un énoncé enchâssant le discours qui est rapporté, on peut supposer que les deux syntagmes : tu continues / tu vas 
au lycée correspondent à l'interaction avec un autre énonciateur que l'on pourrait gloser ainsi : mon oncle m'a dit : après le collège tu continues / tu vas au lycée.

Cette orientation dialogique est récurrente dans les entretiens. Elle manifeste chez l'apprenant la volonté de s'adosser pour sa production à une autre instance énonciative légitimée par le contexte social en amont et surtout validante. Ici, l'énoncé est très certainement issu des discours de son oncle, l'adulte référent qui gère sa situation en France. Le contexte psycho- affectif et social dans lequel s'inscrit le discours est essentiel car il oriente l'énoncé. Cette polyphonie est sans doute un recours linguistique qui marque l'adhésion du locuteur au discours antérieur, avec lequel il semble construire une position dialogique de reprise en écho. Il s'en saisit pour le valider et produire à partir de celui-ci un nouvel énoncé. L'interaction dialogique de ces deux énoncés apparaît alors comme un système dynamique au service de la production langagière.

Par ailleurs, un peu plus loin, l'origine d'un discours rapporté est clairement explicitée par le syntagme : m'a dit tonton. Reprenons ici le passage concerné :

Ibrahim : m'a dit tonton // je dis je rentre / m'a dit: attends les papiers après si / tu tombes ou t'as mal t'as // les papiers//

$\mathrm{P}$ : et oui

Le discours rapporté ici utilise les marques déictiques et modales de ce qui est sûrement l'Énoncé 1 comme l'atteste l'impératif: attends ( $2^{\mathrm{e}}$ personne singulier.). Le locuteur, Ibrahim, explicite clairement le fait que c'est autrui (son oncle) qui porte la responsabilité énonciative des paroles: m'a dit. À ce moment-là de la production langagière, et à contrario de l'exemple précédent, l'apprenant a su trouver les outils linguistiques adéquats pour expliciter l'origine du discours cité. S'agit-il, dans ce second cas, d'une vigilance métalinguistique plus forte ? Dans tous les cas, il s'agit, de fait, d'entretenir une relation dialogique avec un discours d'origine qui résonne en écho, au sein même de son énoncé. En effet, il est important de noter que la discussion entre les deux protagonistes a très certainement eu lieu en arabe, l'oncle ne s'exprimant quasiment pas en français. Le locuteur (Ibrahim) a donc dû procéder à une traduction de l'Énoncé 1 afin d'orienter son énoncé vers l'interlocuteur francophone. La construction d'un discours rapporté direct a été motivée par le besoin d'être compris. Les hésitations entre le je et le tu et les temps d'arrêt au moment de la production langagière témoignent sans doute de la difficulté pour le locuteur de retrouver les déictiques et modalités de l'Énoncé 1. Le locuteurénonciateur (Ibrahim) a probablement recours à cette mise en scène rhétorique comme à une béquille énonciative afin de pallier ses difficultés de construction syntaxique. Le discours direct apparaît ici comme une composante essentielle de sa construction linguistique, en l'occurrence une sorte de raccourci syntaxique.

$\mathrm{P}:$ qu'est-ce que tu veux me raconter $\uparrow$

A : une fois en Italie / je travaille bien et après mon père il m'a donné une moto petite

Dans cet extrait, on rencontre un emploi du présent dans la proposition principale suivi par un temps du passé (passé composé) dans la deuxième proposition. Le présent de l'indicatif: je travaille bien, interroge. En effet il ne situe pas le procès par rapport au moment de l'énonciation, le nunc (temps absolu), mais le situe comme antérieur à l'autre procès (temps relatif) il m'a donné (Gosselin, 2006). Le circonstant temporel : une fois en 
Italie, a en charge de préciser à l'interlocuteur $(\mathrm{P})$ qu'il s'agit d'une instruction temporelle relevant du passé. Or du point de vue de son niveau d'acquisition, l'emploi du passé composé m'a donné atteste du fait que l'apprenant commence à acquérir les formes verbales du passé.

Par ailleurs temporalité et modalité sont deux dimensions essentielles de l'énoncé. Les études linguistiques et notamment les grammaires opposent généralement temporalité et modalité des temps verbaux. Or pour Gosselin, cette dichotomie entre dimension temporelle et modale peut paraitre parfois excessive car ne couvrant pas toutes les réalités des énoncés. En effet ici l'usage du présent semble revêtir une dimension modale car il exprime une attitude du locuteur vis-à-vis de son propre énoncé. Ce présent, enclavé dans des circonstants marquant le passé, évoque une modalité assertive du locuteur. Celui-ci semble réactualiser un présent vécu sans doute avec une forte charge affective : je travaille bien. Le présent serait ici pour le locuteur apprenant un mode de validation de la prédication : travaille bien. D'apparence fautif car n'appartenant pas au hic et nunc de l'échange effectif, il serait la réactualisation d'une autre interaction réalisée dans une autre sphère d'échange et avec laquelle il entretiendrait une relation dialogique de reprise.

\section{Extrait 3}

$\mathrm{P}$ : et ce matin qu'est-ce que tu as fait avec monsieur $\mathrm{R} \uparrow$

Marco : ce matin // on fait des mathématiques des mesures et // géographie

et voilà //

$\mathrm{P}$ : et du sport aujourd'hui $\uparrow$

Marco : oui

$\mathrm{P}:$ quel sport $\uparrow$

Marco : et // et // le maitre y a une batte et une balle on joue a baseball et //

et // ba chercher la balle (Intonation impérative)

$\mathrm{P}:$ va chercher la balle $\uparrow$ qu'est-ce que c'est $\uparrow$ je ne comprends pas

Marco : // le maitre tire // il fait comme dé baseball // il fait avec des battes

dures

et on va chercher

$\mathrm{Au}$ moment de l'enregistrement, Marco est en France depuis 2 mois seulement. Sa langue maternelle est l'espagnol. Malgré les efforts de ses enseignants pour le rassurer, Marco a toujours manifesté beaucoup d'inhibition pour s'exprimer en français. Ces nombreuses hésitations et ses temps de silence, marquent sans doute une forte insécurité linguistique, sans doute d'ordre psychoaffectif. On s'intéressera ici à l'extrait de discours :

ba chercher la balle.

D'un point de vue micro-syntaxique, le déictique utilisé par le biais de l'impératif : ba (va) se réfère à une instance énonciative extérieure. Il y aurait donc dans cet énoncé deux énonciateurs : Marco et son professeur. Or, dans le discours, ces deux énonciations sont traitées sans hiérarchie énonciative marquée. Il n'y a pas de trace de discours rapporté, notamment avec la trace d'un énoncé enchâssant qui pourrait être : le maître a dit. Seule l'intonation reproduite et marquée de l'impératif nous laisse penser qu'il s'agit des paroles du professeur.

24 Ce premier énoncé : ba chercher la // balle, celui du professeur de Marco, a été produit dans un contexte qui, en l'illustrant, sémantise l'énoncé. La production verbale de Marco rentre en interaction dialogique avec ce premier discours qui fonctionne en écho et est intégré tel quel à la nouvelle production langagière. On peut supposer que ce n'est que 
plus tard que l'on trouvera les marques déictiques et modales justes et adéquates pour présenter le premier énoncé. On peut émettre l'hypothèse selon laquelle les situations d'interactions verbales entre l'apprenant et les autres locuteurs-énonciateurs sont une situation stimulante qui facilite la mémorisation de la langue cible. Comme dans les occurrences précédentes, le discours rapporté est fréquemment utilisé pour rapporter des faits passés. L'énoncé du professeur est restitué ici dans toute son intégrité et sa correction syntaxique. Il s'agirait donc d'une étape d'un processus plus global d'acquisition de la langue cible, dont l'écho dialogique serait le levier.

Ainsi nous avons pu analyser des segments discursifs marqués par l'orientation dialogique interdiscursive (Bres, 2005). Pour l'interlocuteur-récepteur, l'énoncé de l'apprenant laisse parfois entendre une autre voix perceptible au travers de ce qui pourrait s'apparenter à des erreurs de construction syntaxique. La trace de cette autre voix ne serait pas alors un vestige inerte, mais un élément bien actif de sa production langagière. Elle porterait la marque d'un autre discours, d'un autre acte de parole. Sa présence matérialiserait le lien, le fil linguistique avec lequel l'apprenant est relié à d'autres instances énonciatives antérieures et avec lesquelles il construit le sens de ses discours.

\section{Conclusion}

A travers les quelques exemples analysés ici, mettant en lumière l'usage spécifique du présent ou le référentiel variable des déictiques, nous avons tenté de mettre en évidence la dimension subjective et intersubjective mise en jeu dans les processus d'apprentissage des sujets de ce corpus. L'expression extérieure du discours telle que la définit Bakhtine est la dernière étape d'un long processus ayant eu lieu en amont et pendant lequel le discours antérieur a été façonné. Durant toute une année scolaire, leur production langagière a été observée et analysée. Nous avons ainsi tenté de mettre à jour les orientations diverses des énoncés et la polyphonie qui les compose. L'expression extérieure se révèle être le résultat de divers réseaux d'influences, absorbés par les locuteurs dans leur volonté de communiquer. Ainsi, l'analyse de leurs discours dans l'interaction verbale a mis en lumière la dimension dialogique des énoncés comme une composante incontournable de la construction syntaxique des apprenants. De façon récurrente, le discours produit est le résultat d'un enchevêtrement de voix dont les marques linguistiques restent floues - qui parle? - tant elles sont imbriquées les unes aux autres. Ces autres discours auxquels le sujet se réfère au travers du procédé dialogique interdiscursif constituent le socle et le fondement de la construction du nouvel énoncé. Dans cet univers linguistique plurilingue où l'on retrouve simultanément langue maternelle, langues secondes et français, les hésitations, les tâtonnements verbaux de l'apprenant (reformulations, temps de silence, emprunts aux langues familiales) manifestent sans doute la déstabilisation produite par ce choc linguistique. Cependant l'expression extérieure incarnée dans l'activité langagière se trouve dynamisée. Son développement semble assujetti à ces différentes interactions verbales mais aussi sociales. Il est ainsi nettement apparu sur l'ensemble des sujets du corpus que le cadre social tel que le définit Bakhtine, c'est-à-dire la situation sociale la plus immédiate (1929-1977: 123) dans laquelle était inscrit l'acte de parole, constituait pour l'apprenant un cadre sémantique. L'énoncé subit l'éclairage de cet environnement social qui souvent oriente la compréhension. Ainsi, les choix linguistiques opérés, utilisation du discours rapporté (extraits 2 et 3), recours ou emprunts aux langues 
familiales, ne sont que la manifestation de l'orientation du discours vers un auditoire social comme l'atteste la dimension interlocutive du dialogisme. Cet auditoire est un prisme concret qui permet à l'apprenant d'inscrire son discours dans un territoire connu et clairement défini. Il semblerait que la nécessité de communiquer engage le locuteur à construire une position dialogique avec cet environnement-là, au travers d'énoncés passés ou à venir. Ainsi, et de façon plus globale, en analysant au plus près, au sein même des formes micro-syntaxiques, le parler de ces jeunes migrants, nous avons essayé de mettre en évidence le lien étroit qu'entretenait l'interaction verbale et le dialogisme défini par Bakhtine-Volochinov.

Ainsi donc, la notion de dialogisme apparait opératoire lorsqu'il s'agit d'analyser des situations d'oral conversationnel en situation d'interlangue. En prenant en compte la dimension sociale et idéologique de l'énoncé, au sens Bakhtinien du terme, le chercheur est amené à retrouver certains aspects de la vie du discours qui participent à l'organisation formelle de celui-ci au niveau syntaxique, ou morphosyntaxique, et lexical. Le dialogisme est un processus dynamique mis en œuvre dans l'apprentissage et qui va au-delà des spécificités linguistiques propres à chaque langue. Dans l'espace interlinguistique dans lequel évolue le jeune apprenant, le dialogisme apparaît comme un composant incontournable de l'acte de parole qui transcende les différents univers linguistiques : langues d'origine et langue cible. Les langues se parlent, dialoguent entre elles, s'interpellent et se répondent. Le dialogisme gère cet espace d'acquisition en fournissant, au travers de matériaux discursifs, cette énergie nécessaire à la création d'un nouvel énoncé, première étape de la construction de la langue cible.

\section{BIBLIOGRAPHIE}

Anscombre, J.-Cl. (2006). « Stéréotypes, gnomicité, et polyphonie : la voix de son maître. In Perrin (dir.). Le sens et ses voix. Dialogisme et polyphonie en langue et en discours. Recherches linguistiques, $\mathrm{n}^{\circ}$ 28. Université Paul Verlaine, Metz. pp. 349-378.

Ben Amor Ben Hamida, T. (2009). «Erreurs interférentielles arabe-français et enseignement du français ». Synergie Tunisie, $\mathrm{n}^{\circ} 1$. pp. 105-117.

Bakhtine, M. \& Volochinov, V. (1929-77). Le marxisme et la philosophie du langage. Paris : Minuit.

Bres, J. \& Mellet, S. (2009). Dialogisme et marqueurs grammaticaux. Langue française $n^{\circ} 163$, pp. 3-20.

Bres, J., Haille, P., Mellet, S., Nølke, H. \& Rosier, L. (éds.). (2005). Dialogisme, polyphonie : approches linguistiques. Actes du colloque de Cerisy, 2004. www.ccic-cerisy.asso.fr/dialogismeTM05.html.

Bronckart, J.-P. (1997). Activité langagière, textes et discours. Pour un interactionnisme socio-discursif. Paris : Delachaux et Niestlé.

Du Bois, J. W. (2014). Towards a dialogic syntax. Cognitive Linguistics, $\mathrm{n}^{\circ} 25$ (3). University of California. Santa Barbara. pp. 359-410. 
Gosselin, L. (2006). « De la distinction entre la dimension temporelle de la modalité et la dimension modale de la temporalité ». Cahiers de Praxématique, n47. pp. 21-52.

Moirand, S. (2004). Le dialogisme entre problématiques énonciatives et théories discursives Cahiers de praxématique, $\mathrm{n}^{\circ} 43$. pp.189-217.

Maingueneau. D. (2012). Analyser les textes de communication. Paris : Armand Colin.

Maingueneau, D. (1996). Aborder la linguistique. Paris : Seuil.

Naddaf, N. (1993). Etude comparative des deux systèmes linguistiques du français et de l'arabe et problèmes d'interférences. Université de Lattaquié Syrie : Document CNRS.

Petit, J. (2001). « Le bilinguisme précoce et ses avantages ». http://christian.huber.pagespersoorange.fr/bilinguismebis.htm.

Porquier, R. (1994). «Communication exolingue et contextes d'appropriation : le continuum acquisition/apprentissage ». Py, B. (dir.) L'acquisition d'une langue seconde. Quelques développements théoriques récents. Bulletin VALS/ASLA, n59. pp.159-170.

Porquier, R. (1981). « Remarques sur les interlangues et leurs descriptions ». Etudes de linguistique appliquée, $\mathrm{n}^{\circ}$ 63. pp. 7-17.

Py, B. (2002). « Acquisition d'une langue seconde, organisation macrosyntaxique et émergence d'une microsyntaxe ». Marges linguistiques, n³. pp. 48-55.

Py, B. (2000). « Didactique des langues étrangères et recherche sur l'acquisition. Les conditions d'un dialogue ». Études de Linguistique Appliquée, n¹20. pp. 395-404.

Sauvage, J. (2012). L'enfant et le langage : Une approche dynamique et développementale. Paris : L'Harmattan.

Sauvage, J. \& Guiraud, F. (2015). « Acquisition d'une récursivité syntaxique en français langue seconde : Exemple du pronom relatif ». Carnets d'Atelier de Sociolinguistique, n ${ }^{\circ}$. pp. 103-116.

Sauvage, J. (2015). L'acquisition du langage. Un système complexe. Paris : L'Harmattan.

Selinker, H. (2014). « Interlanguage 40 years on : three themes from here ». In ZhaoHong \& Tarone (eds.) Interlanguage. Forty years later. Amsterdam/Philadelphia : John Benjamins Publishing Compagny. pp. 223-224.

Véronique, D. (2009). L'acquisition de la grammaire du français langue étrangère. Paris : Didier.

\section{NOTES}

1. Convention de transcription: / pause brève, // pause longue, $\uparrow$ intonation montante.

\section{RÉSUMÉS}

Nous proposons dans cet article d'étudier la production langagière, en contexte scolaire, de jeunes migrants apprenants du français. Pour analyser le discours de ces apprenants, le cadre 
théorique du dialogisme apparaît opératoire notamment dans sa dimension interdiscursive. En effet, l'observation de la matérialité discursive de leurs énoncés au niveau microsyntaxique révèle l'emploi récurrent de constructions syntaxiques spécifiques à ce type de situation de communication. Pour l'interlocuteur-récepteur, l'énoncé de l'apprenant laisse parfois entendre une autre voix perceptible au travers de ce qui pourrait s'apparenter à des erreurs de construction syntaxique. Nous pourrons ainsi constater que le dialogisme semble gérer un espace interlinguistique dans lequel se situe le discours de l'apprenant. Nous analyserons ainsi, les emplois spécifiques des pronoms déictiques et des temps verbaux (présent). Nous verrons que le cadre dialogique tel qu'il est défini par Bakhtine-Volochinov (1929-1977) peut nous permettre de formuler quelques hypothèses explicatives sur ce qui pourrait apparaitre comme des emplois fautifs.

Our purpose in this article is to study language production in a school environment for young migrants learning French as a foreign language. To analyze the discourse of these learners, the theoretical framework of dialogism appears operative in particular in its interdiscursive dimension. Indeed, the observation of the discursive materiality of their utterances at the microsyntactic level reveals the recurrent use of specific syntactic construction. For the interlocutor-receiver the utterance of the learner sometimes implies another perceptible voice through what might resemble syntactic construction errors As a matter of fact their utterances belong to an interlinguistic space which seems managed by dialogism. We will hereby focus on the specific use of deictic pronouns and verbal tenses. We will see that the dialogic context such as defined by Bakhtine-Volochinov (1929-1977) may enable us to formulate hypotheses and explanations as to what might be considered incorrect use of the language.

\section{INDEX}

Mots-clés : dialogisme, espace interlinguistique, apprenants du français, analyse de discours Keywords : dialogism, interlinguistic space, french learners, discourse analysis

\section{AUTEUR}

\section{FLORENCE GUIRAUD}

Université Paul-Valéry, Montpellier 3 - laboratoire Praxiling - UMR 5267

Florence Guiraud est professeur des écoles, elle enseigne le Français Langue Seconde en classe d'accueil pour élèves allophones (UPE2A) à Sète. Elle est aussi chargée de cours à l'Université Paul-Valéry, Montpellier 3. Sa thèse de doctorat en cours porte sur l'étude de l'espace interlinguistique chez les apprenants du français en contexte scolaire : aspects sociointeractionnistes, dialogiques et polyphoniques (dir. J. Sauvage).

florence.guiraud [at] wanadoo.fr 